Rabaska

Revue d'ethnologie de l'Amérique française

GAUTHIER, ANTOINE. La Sculpture d'art populaire. Québec, Conseil québécois du patrimoine vivant, " Les traditions culturelles du Québec en chiffres ", vol. 3, 2018, 60 p. ISBN 978-2-922180-25-1

\title{
Jean-François Blanchette
}

Volume 17, 2019

URI : https://id.erudit.org/iderudit/1066029ar

DOI : https://doi.org/10.7202/1066029ar

Aller au sommaire du numéro

Éditeur(s)

Société québécoise d'ethnologie

ISSN

1703-7433 (imprimé)

1916-7350 (numérique)

Découvrir la revue

Citer ce compte rendu

Blanchette, J.-F. (2019). Compte rendu de [GAUTHIER, Antoine. La Sculpture d'art populaire. Québec, Conseil québécois du patrimoine vivant, « Les traditions culturelles du Québec en chiffres », vol. 3, 2018, 60 p.

ISBN 978-2-922180-25-1]. Rabaska, 17, 301-305.

https://doi.org/10.7202/1066029ar d'utilisation que vous pouvez consulter en ligne. 
père, Franz, et qu'il a reproduit en exergue dans Do Kamo : « Ils te diront peut-être des choses étranges, mais écoute d'abord, et tâche de comprendre en traduisant ce qu'ils te disent dans ta mentalité : tu verras peut-être alors que ce n'est pas si étrange, mais seulement dans une autre langue que celle qui correspond à notre mentalité... » (24 décembre 1902). Voilà !

Bertrand Bergeron

Saint-Bruno en Lac-Saint-Jean

Gauthier, Antorne. La Sculpture d'art populaire. Québec, Conseil québécois du patrimoine vivant, «Les traditions culturelles du Québec en chiffres », vol. 3, 2018, 60 p. ISBN 978-2-922180-25-1.

Dans cette étude qu'il réalise au nom du Conseil québécois du patrimoine vivant (CQPV), l'auteur désire jeter un nouveau regard sur notre compréhension de l'art populaire au Québec grâce aux résultats d'une analyse statistique. Il choisit à cet effet la catégorie des sculpteurs, mais il n'explique pas ce choix. Deux corpus servent à son analyse, d'une part les réponses aux questionnaires venant de sculpteurs se comptant eux-mêmes comme artistes populaires et, d'autre part, celles venant d'organismes intéressés par le sujet. L'auteur désire de plus en tirer des pistes de développement afin de mieux faire connaître l'art populaire et améliorer le sort des artistes qui pratiquent cette forme d'art.

Dès son introduction, l'auteur annonce : « Les résultats du présent rapport représentent mutatis mutandis des indications utiles pour une compréhension améliorée de l'art populaire et de sa (ou ses) définition(s) (p. 6).

Il aurait été majeur de bien définir, à la base de cette étude, le groupe qui en est l'objet et ensuite de s'assurer que l'échantillon qu'on utilise le représente bien. Comme on n'a pas de définition claire de qui est considéré comme un sculpteur en art populaire, il est bien difficile de penser qu'on a pu obtenir un échantillon représentatif. De plus, le chapitre 1, qui quantifie les acteurs et leur pratique, est basé sur 47 personnes, alors qu'on pense qu'il y en aurait peut-être 750 au Québec (p. 10). L'auteur croit que cette approche quantitative viendra surpasser les approches qualitatives utilisées par d'autres, mais, chose surprenante, il fait plusieurs mises en garde contre toute interprétation basée sur ce nombre limité de participants... Il procède malgré tout et tire de cet échantillon extrêmement faible toute une série de statistiques, illustrées de 28 figures et quelques tableaux.

L'auteur évite de définir lui-même l'art populaire et il indique qu'il laisse cela aux « principaux acteurs de ce sous-secteur» (p. 6) ou encore, ce qui n'est pas la même chose, « aux divers acteurs du secteur » (p. 56). J'imagine que dans le premier cas, « les principaux acteurs » sont les sculpteurs 
eux-mêmes. Si cela était, il s'agirait d'une approche originale qui n'a pas été utilisée auparavant dans des études en art populaire, à ma connaissance. Cette approche aurait l'avantage de laisser aux pratiquants le soin de se définir eux-mêmes. Mais ce n'est pas finalement ce que fait l'auteur, comme il l'indique lui-même en présentant 18 énoncés sur l'art populaire ou sur la sculpture d'art populaire, qu'il résume ainsi : «Cette annexe présente un échantillon des réponses obtenues grâce aux différents questionnaires de l'enquête qui donne un aperçu de la définition de l'art populaire fournie par les divers acteurs du secteur. Elles ont notamment été sélectionnées pour leur caractère représentatif sans toutefois fournir un inventaire exhaustif. On remarque en outre l'importance des qualificatifs d'autodidacte, du peuple, des thèmes liés au quotidien ou à la vie traditionnelle (histoire), de l'aspect recyclage \& récupération et de la difficile reconnaissance de cet art. » (p. 56)

Or, ces réponses ne proviennent pas seulement des sculpteurs, mais des « divers acteurs du secteur », soit les sculpteurs, les muséologues, les galeristes, les propriétaires de boutiques, les antiquaires et les organisateurs de festivals. L'objectif de départ n'est donc pas atteint puisque d'autres intervenants que les sculpteurs ont eu voix à s'exprimer sur l'art populaire dans cette annexe erronément intitulée « Définitions de l'art ou de la sculpture d'art populaire ».

Au niveau de la définition d'un sculpteur en art populaire, on a demandé aux 47 sculpteurs qui ont été retenus pour cette étude de cocher les diverses appellations dans lesquelles ils se reconnaissaient. On a obtenu les résultats suivants : sculpteur en art populaire (près de $75 \%$ ), gosseux (41\%), patenteux (5\%), chef d'œuvreux (5\%), artiste (50\%), artisan (35\%), autres $5 \%$ (p. 13). Je trouve intéressant de voir la variété des étiquettes sous lesquelles les sculpteurs de cette étude aiment se retrouver. D'autant plus que le concept d'art populaire n'a pas été créé par les artistes en art populaire. Ce concept a été développé à partir d'observations faites sur le terrain par les ethnologues ou les collectionneurs qui ont voulu identifier une forme de pratique qui n'était pas reconnue, ni valorisée par les beaux-arts. Mon observation est qu'un nombre limité d'artistes en art populaire utilisent cette appellation spontanément pour se définir. Comme Clémence Lessard, l'une des artistes populaires bien connue et présente dans les festivals en art populaire dont il est question dans l'étude, me mentionnait : «Autrefois on me disait que je faisais de l'art naïf. Aujourd'hui on me dit que je fais de l'art populaire. Je n'aime pas plus cela. » D'ailleurs 12 sculpteurs sur les 47 (25\%) n'ont pas coché la catégorie « sculpteur en art populaire ».

Au niveau de la définition de la sculpture en art populaire, l'auteur affirme que la nature de l'apprentissage de cette pratique est intimement liée à la définition même de l'art populaire. Comme un seul sculpteur de cette étude 
tient sa pratique d'un parent et que $82 \%$ des répondants indiquent être autodidactes, l'auteur écrit que cette dernière caractéristique « semble se situer au cœur même de la notion d'art populaire » et il ajoute par ailleurs dans le même paragraphe qu'elle fait partie du paradigme particulier de cette forme d'art (p. 13). Par contre, dans un argument qu'il présente plus loin en faveur de la reconnaissance de la sculpture en art populaire au patrimoine immatériel du Québec, il écrit qu'il ne comprend pas le refus du ministère de la Culture et des communications qui conclut que « la pratique ne remplit pas le critère de "transmission de génération en génération" " (p. 44). Pourtant son étude contredit son propre argument et concourt plutôt à l'avis du ministère.

Toujours au niveau de la définition de la sculpture en art populaire, au chapitre 2 qui traite des établissements qui s'intéressent à l'art populaire et aux événements qui en font la promotion, l'auteur considère clairement que les produits suivants ne font pas partie de ce domaine : les vire-vent et girouettes, les bas-reliefs, les maquettes de villages, les meubles sculptés, l'art populaire religieux, les objets décoratifs divers, les jeux et les appelants (p. 29 et p. 33). Cela limite donc le domaine de la sculpture en art populaire à une catégorie qui demeure obscure puisqu'elle n'est pas définie. Tout le chapitre 2 où « l'action des divers festivals et expos-ventes de même que celle des musées y est quantifiée » (p. 7) est donc invalidée par l'absence d'une définition claire et l'exclusion de nombreuses œuvres que plusieurs spécialistes en art populaire considèrent comme de l'art populaire.

Au sujet de la pratique, on découvre que les 47 sculpteurs de ce groupe ont tous commencé à sculpter avant la retraite, ce qui amène l'auteur à conclure : «Sculpter ne représente en sorte pas un passe-temps [...] Cela représentera plutôt une activité artistique à part entière développée à l'âge économique actif et poursuivie, lorsqu'applicable, à la retraite » (p. 11). Plus loin, il indique que le temps consacré à cette pratique est limité pour plusieurs d'entre eux. Ainsi, 8 sculpteurs y consacrent 5 heures et moins par semaine, 5 entre 6 et 10 heures, 9 entre 11 et 20 heures, 13 entre 21 et 40 heures et 4 plus de 40 heures (p. 14). Il s'agit donc, pour la majorité, d'une activité pratiquée à temps partiel. Au point de vue économique, $89 \%$ des répondants indiquent qu'ils doivent faire un autre travail pour subvenir à leurs besoins (p. 23), quatre n'en tirant aucun revenu et 13 , sur les 30 qui ont répondu à cette question, n'en tirant que d'un à mille dollars (p. 22). L'auteur conclut : « Il s'agit donc d'un secteur professionnel la plupart du temps atypique et générant peu de revenus totaux » (p. 22). En somme, bien que l'auteur considère que l'art populaire est une profession et bien qu'il clame que « le milieu socioéconomique des sculpteurs en art populaire répondants ne semble pas différer de façon significative de celui des autres artistes en arts visuels » (p. 52), les résultats de 
son enquête révèlent plutôt qu'être sculpteur en art populaire n'est pas une profession en soi et qu'il s'agit d'une occupation économique secondaire.

Dans sa conclusion, l'auteur reprend les diverses caractéristiques qui ont été utilisées par le passé pour qualifier et définir l'art populaire. Il oppose les travaux faits par les ethnologues avec les festivals récents en art populaire : «À partir d'une situation dans laquelle des ethnologues et des collectionneurs pionniers établissaient une certaine médiation avec le public - ou à tout le moins avec l'université -, à partir d'une situation donc dans laquelle des représentants d'institutions accordaient pour la première fois une importance à ce champ culturel sous l'angle principal de la recherche, nous sommes éventuellement entrés dans une seconde phase où des événements invitent désormais des sculpteurs à se présenter directement au public, dans une phase où des entités associatives comprennent de tels artistes au sein de leur conseil d'administration ou de leur direction artistique, et dans laquelle les artistes ou les artisans ont la possibilité de faire valoir eux-mêmes leur travail grâce aux technologies de l'information. » (p. 52)

Je ne dirais pas que l'auteur est de mauvaise foi, mais il prétend ignorer que les études des ethnologues sur l'art populaire ont toujours été basées sur des enquêtes de terrains, souvent très exhaustives. Par ailleurs, en tant qu'anthropologue et muséologue, j'ai moi-même participé activement aux deux festivals que l'auteur mentionne et je suis même le cofondateur du Festival Les gosseux s'exposent qui s'appelle désormais Le Rendez-vous des sculpteurs en art populaire. Les artistes en art populaire savent tout le respect que j'ai pour eux. Quand j'ai proposé le nom humoristique « Les gosseux s'exposent ", c'était justement pour utiliser une appellation que plusieurs d'entre eux se donnent et les distinguer clairement des artistes des beaux-arts qui font maintenant de l'art populaire. Par ailleurs, la Société québécoise d'ethnologie a été un partenaire de ce festival dès le début, comme l'indique le logo de cette société sur ses programmes. L'intention était de donner une avenue aux artistes afin de présenter leurs œuvres au grand public. Enfin, l'auteur de l'étude va bien au-delà de la réalité quand il indique que «nous sommes éventuellement entrés dans une seconde phase [...] où des entités associatives comprennent de tels artistes [sculpteurs] au sein de leur conseil d'administration ou de leur direction artistique ». Le Rendez-vous des sculpteurs en art populaire se tient sur invitation et n'a ni conseil d'administration, ni sculpteur à la direction artistique. À ce que je sache, la participation des artistes à l'organisation du Festival de sculpture d'art populaire de Saint-Ulric de Matane n'est pas différente.

Enfin, que penser de l'argumentaire de l'auteur? Les objectifs de l'étude sont présentés tout au long de l'ouvrage comme des résultats concluants alors qu'ils ne composent qu'une envolée oratoire qui aurait mérité une assise 
solide pour soutenir ses prétentions. Quant aux nombreux chiffres qui sont au centre de ce projet statistique, il est approprié de citer l'auteur lui-même : «Les données de sondage présentées sont des résultats bruts n'ayant pas fait l'objet de traitement statistique pour les rendre représentatifs de la population d'artistes et d'intervenants à laquelle appartiennent les sondés » (p. 10).

En résumé, l'auteur a une vision personnelle de la sculpture en art populaire qui ne correspond ni à la réalité qu'il a observée, ni à la réalité présente sur le terrain, pas plus qu'aux nombreux chiffres avec lesquels il jongle.

Jean-François Blanchette

Société québécoise d'ethnologie

Guilbeault-Cayer, Émilie. Les Scurs de la Charité de Saint-Louis en Amérique, 1902-2018. Québec, Septentrion, 2018, 296 p. ISBN 978-28944-8979-6.

Au Québec comme dans plusieurs États à l'échelle internationale, les communautés religieuses traversent actuellement une étape, pour ainsi dire, inédite de leur histoire qui est marquée par la baisse drastique de leurs effectifs. Une telle situation amène un certain nombre de congrégations - étonnamment pas toutes -, à déployer une démarche visant la " pérennisation 》 de leur patrimoine. Une telle démarche est susceptible de revêtir de multiples formes, par exemple la conversion de la propriété conventuelle dans une perspective évolutive et ouverte à l'adoption de nouveaux usages en phase avec la nature du charisme et l'esprit des lieux. La production historiographique peut également servir une démarche de pérennisation du patrimoine des congrégations religieuses. C'est exactement le rôle que joue le présent ouvrage, produit par Émilie Guilbeault-Cayer, détentrice d'une maîtrise en histoire et consultante dans la même discipline. L'auteure ne cherche pas à le dissimuler et l'introduction se veut très explicite à cet égard : " les Sœurs de la Charité de Saint-Louis ont également le désir de faire œuvre utile avec ce récit : contribuer à la connaissance plus large du Québec et du Canada, retracer leur parcours afin de dessiner les contours de leur présence, de leur expansion et de leur rayonnement hors du pays » (p. 15-16).

La perspective monographique qui sous-tend l'ouvrage est très riche en matière factuelle et il faut en féliciter l'auteure qui navigue admirablement à travers l'histoire d'une congrégation arrivée, comme tant d'autres, au Canada français dans le contexte trouble des législations françaises du début du $\mathrm{XX}^{\mathrm{e}}$ siècle, mais qui a su développer son activité dans plusieurs champs notables. Ainsi, l'ouvrage rappelle-t-il à profit, des faits auront peut-être échappé à certains, mais qui sont non moins dignes de mention pour autant, 